PoS $\quad \begin{aligned} & \text { PROCEEDINGS } \\ & \text { OF SCIENCE }\end{aligned}$

\title{
Electroweak Physics at the FCC-ee
}

\section{Tadeusz Lesiak*}

The H.Niewodniczański Institute of Nuclear Physics

Polish Academy of Sciences, Kraków

E-mail: Tadeusz.Lesiak@ifj.edu.pl

The perspectives of precise measurements of electroweak observables at the the Future Circular Collider with $e^{+} e^{-}$beams (FCC-ee) are briefly discussed.

The 39th International Conference on High Energy Physics (ICHEP2018)

4-11 July, 2018

Seoul, Korea

* Speaker.

${ }^{\dagger}$ On behalf of the FCC Design Study Group 


\section{Introduction}

The physics program realized at LEP accelerator [1] comprised a detailed test of the Standard Model of electroweak interactions which was passed with flying colours. In view of the Higgs boson discovery [2] in 2012, even more precise determination of electroweak observables has become one of the primordial goals of particle physics. The latter, together with recent advances in accelerator technologies has reinvigorated the interest in circular electron-positron colliders. Two projects of such accelerators have been proposed in the recent years: the Circular Electron Positron Collider (CEPC) [3] in China and Future Cicular Collider (FCC) [4] at CERN. The FCC design study assumes the construction and exploitation of a $\sim 100 \mathrm{~km}$ tunnel infrastructure in the Geneva area. to host a giant, $100 \mathrm{TeV}$ proton-proton collider as the flagship part of the project. However, the commissioning of the $e^{+} e^{-}$collider, so-called FCC-ee, is envisioned as the first step. This high-luminosity accelerator would span the range of centre-of-mass $(\mathrm{CM})$ energies from below of the $Z$ pole, through the $W W$ and $H Z$ threshold, till the $t \bar{t}$ threshold and above. The FCC-ee collider will profit from recent innovations implemented at $b$-factories (nanobeam scheme, continuous injection, crab-crossing collisions etc.) and its most relevant parameters [7] are presented in Table 1 and compared with LEP phase-2 (LEP2) collider [1].

\begin{tabular}{l|c|cccc}
\multicolumn{1}{c|}{ Parameter } & LEP2 & \multicolumn{4}{c}{ FCC-ee } \\
\hline \hline$E_{b}[\mathrm{GeV}]$ & 104 & 45.6 & 80 & 120 & 182.5 \\
$I[\mathrm{~mA}]$ & 4 & 1390 & 147 & 29 & 5.4 \\
\# of bunches/beam & 4 & 16640 & 2000 & 328 & 48 \\
$\beta_{x}^{*}[\mathrm{~m}]$ & 1.5 & 0.15 & 0.2 & 0.3 & 1.0 \\
$\beta_{y}^{*}[\mathrm{~mm}]$ & 50 & 0.8 & 1 & 1 & 1.6 \\
$\varepsilon_{x}[\mathrm{~nm}]$ & $30-50$ & 0.27 & 0.84 & 0.63 & 1.46 \\
$\varepsilon_{y}[\mathrm{pm}]$ & 250 & 1 & 1.7 & 1.3 & 2.9 \\
$P_{\mathrm{SR}}[\mathrm{MW}]$ & 22 & 100 & 100 & 100 & 100 \\
\hline$L\left[10^{34} \mathrm{~cm}^{-2} \mathrm{~s}^{-1} / \mathrm{IP}\right]$ & 0.012 & 230 & 28 & 8.5 & 1.55 \\
\hline Statistics & & $\sim 5 \times 10^{12} Z$ & $\sim 3 \times 10^{7} W W$ & $\sim 10^{6} \mathrm{ZH}$ & $\sim 10^{6} t \bar{t}$ \\
$(2$ expts $)$ & & $(4$ years $)$ & $(2$ years $)$ & $(3$ years $)$ & $(5$ years $)$
\end{tabular}

Table 1: Selected parameters of the FCC-ee accelerator. The characteristics of LEP2 collider have been supplemented for comparison. The following parameters are presented: beam energy $\left(E_{b}\right)$, beam current (I), number of bunches, horizontal (vertical) beta function at the interaction point (IP) $\beta_{x}^{*}\left(\beta_{y}^{*}\right)$, horizontal (vertical) emittance $\left(\varepsilon_{x}\left(\varepsilon_{y}\right)\right)$, synchrotron radiation power $\left(P_{\mathrm{SR}}\right)$, luminosity $(L)$ and expected statistics of relevant events (from [6].

In the next two chapters the precision electroweak measurements around the $Z$ pole and $W W$ production threshold are briefly discussed.

\section{Electroweak precision measurements at the $Z$ pole}

The FCC-ee offers a very attractive opportunity to go back to the electroweak physics studies at the $Z$ pole. This time, however, the expected statistics of $5 \times 10^{12} Z$ events (cf. Table 1), 
would correspond to the increase by five orders of magnitude to compare with LEP1. As a result, the overall precision of electroweak observables will be generally limited by systematic uncertainties. Special attention should be thus paid to the reduction of them. The accuracy of CM energy calibration, realized via resonant depolarisation will be of particular importance. It would lead to the systematic error of $\leq 100 \mathrm{keV}$ on both the mass and width of the $Z$ boson to compare with $\Delta M_{Z}=2.1 \mathrm{MeV}$ and $\Delta \Gamma_{Z}=2.3 \mathrm{MeV}$ at LEP1. Correspondingly, the gain in precision by actor of 20 is envisioned for the evaluation of the normalized partial widths $R_{l}=\Gamma_{\text {had }} / \Gamma_{l \bar{l}}, l=e, \mu, \tau$ and $R_{q}=\Gamma_{q \bar{q}} / \Gamma_{\text {had }}, q=b, c$ ( $\Gamma_{\text {had }}$ is the total hadronic width). The FCC-ee electroweak physics program at the $Z$ pole envisions also new measurements of the relevant set of forward-backward and polarisation asymmetries. Among them, the $\tau$ lepton pairs offer a unique option of measuring also the polarisation of the final state fermion through the angular distributions and momenta of the decay products. The asymmetries, together with $Z$ partial widths, allow for an extraction of the vector $\left(g_{V}^{f}\right)$ and axial $\left(g_{A}^{f}\right)$ couplings of the neutral current to fermions with the expected relative precisions collected in Table 2 [8]. Finally, the effective Weinberg electroweak mixing angle, defined as: $\sin ^{2} \theta_{W, \text { eff }}=1 / 4\left(1-g_{V}^{f} / g_{A}^{f}\right)$, can be determined with the gain in the precision of 75 (100) for the tau polarisation (muon forward-backward asymmetry) measurements, respectively.

\begin{tabular}{c|c|c|c|c|c} 
lepton & $\Delta g_{V}$ & $\Delta g_{A}$ & quark & $\Delta g_{V}$ & $\Delta g_{A}$ \\
\hline$e$ & $2.5 \times 10^{-4}$ & $1.5 \times 10^{-4}$ & $b$ & $1.0 \times 10^{-2}$ & $1.5 \times 10^{-3}$ \\
$\mu$ & $2.0 \times 10^{-4}$ & $2.5 \times 10^{-5}$ & $c$ & $1.0 \times 10^{-2}$ & $2.0 \times 10^{-3}$ \\
$\tau$ & $3.5 \times 10^{-4}$ & $0.5 \times 10^{-4}$ & & &
\end{tabular}

Table 2: The relative uncertainties on fermion vector and axial neutral couplings to $Z$.

The formidable experimental precision on the $A_{F B}^{\mu \mu}$ allows also for a precise, direct determination of the electromagnetic coupling constant at the $Z$ mass scale $\left(\alpha_{\mathrm{QED}}\left(m_{Z}^{2}\right)\right)$. As demonstrated in [9], the relative uncertainty of the $\alpha_{\mathrm{QED}}\left(m_{Z}^{2}\right)$ is proportional to the one of $A_{F B}^{\mu \mu}$. By taking the data in two one-year periods of time in the optimal working points at $\sqrt{s_{-}}=87.9 \mathrm{GeV}$ and $\sqrt{s_{+}}=94.3 \mathrm{GeV}$, the electromagnetic coupling constant at the $Z$ mass can be determined, without the necessity of extrapolation from low mass region, with the relative precision of $3 \times 10^{-5}$. This value is adequate for future precision electroweak physics fits.

The FCC-ee can also address the question of the number of light neutrino species by measuring the $Z$ invisible width $\left(\Gamma_{\text {inv }}\right)$. The first approach will be based on the determination of the hadronic cross section at the $Z$ peak $\left(\sigma_{\text {had }}^{\text {peak }, 0}\right)$ :

$$
N_{v}=\left(\frac{\Gamma_{l \bar{l}}}{\Gamma_{v} \bar{v}}\right)_{\mathrm{SM}} \cdot\left(\sqrt{\frac{12 \pi R_{l}}{M_{Z}^{2} \sigma_{\text {had }}^{\text {peak }, 0}}}-R_{l}-3\right)
$$

and its accuracy is expected to be of 0.0008 (stat.) \pm 0.001 (syst.) (cf. the LEP1 result $N_{v}=2.991 \pm$ 0.007 [10]). The second approach would exploit the radiative return process $e^{+} e^{-} \rightarrow Z \gamma, Z \rightarrow$ $v \bar{v}$ measured at $\mathrm{CM}$ energies above the $Z$ peak. The number of light neutrino species will de determined from the formula

$$
N_{v}=\left(\frac{e^{+} e^{-} \rightarrow \gamma Z_{\text {inv }}}{e^{+} e^{-} \rightarrow \gamma Z_{l \bar{l}}}\right)^{\text {meas }} /\left(\frac{\Gamma_{\nu \bar{v}}}{\Gamma_{l \bar{l}}}\right)^{\mathrm{SM}} .
$$


Due to the normalization to the number of events observed in the final state $l \bar{l} \gamma$, the systematic uncertainties are relatively small and the expected statistical accuracy amounts to $\times 10^{-3}$ (LEP: $2.92 \pm 0.05[10])$.

\section{Electroweak physics at the $W W$ production threshold}

The FCC-ee aims also at the collection of an enormous sample of $W W$ pairs $\left(3 \times 10^{7}\right.$ events, at and above the production threshold) [8], which would allow for precise measurements of $W$ boson properties, together with trilinear gauge couplices (TGCs) related to the $W W Z$ and $W W \gamma$ vertices.

The mass and width of the $W$ boson will be extracted from the $W W$ cross-section scan in the close range of the production threshold. The expected precisions are $\Delta M_{W}=1 \mathrm{MeV}$ and $\Delta \Gamma_{W}=1.5 \mathrm{MeV}$. The determination of $W$ branching ratios to hadrons and $l \bar{v}, l=e, \mu, \tau$ will yield important tests of the lepton and lepton-quark universalities, with the accuracy below the permill level to compare with current precision of few percent. The limits TGCs are expected to be improved by a factor of 50 .

\section{Summary and Acknowledgements}

The FCC-ee project offers a vast physics programme, in particular in the sector of electroweak observables. Few selected topics from this field have been briefly discussed.

I would like to thank Alain Blondel for his help in preparing the talk. This research was supported in part by grant of Narodowe Centrum Nauki 2016/23/B/ST2/03927.

\section{References}

[1] A.Straessner, "Electroweak Physics at LEP and LHC", Springer, 2010.

[2] A. Nisati and V. Sharma, "Discovery of the Higgs Boson", World Scientific, 2017.

[3] http://cepc.ihep.ac.cn

[4] http://fcc.web.cern.ch

[5] "CERN thinks bigger", CERN Courier magazine, June 2018, https: cerncourier.com/cern-thinks-bigger/

[6] F. Bordry et al., Machine Parameters and Projected Luminosity Performance of Proposed Future Colliders at CERN, CERN-ACC-2018-0037.

[7] http://tlep.web.cern.ch/content/machine-parameters

[8] R. Tenchini, talk at the 2nd FCC Physics Workshop, Jan. 2018, https://indico.cern.ch/event/618254/contributions/2833226/attachments/1582780/2501324/FCCphysicsweek2-zpole-precision.pdf

[9] P. Janot, Direct measurement of $\alpha_{\mathrm{QED}}\left(m_{Z}^{2}\right)$ at the FCC-ee, JHEP 02 (2016) 053.

[10] M.Tabanashi et al. (Particle Data Group), Phys. Rev. D98, (2018) 030001.

[11] A. Blondel et al., [hep-ex/1411.5230]. 